\title{
The Hand Mirror Image: Which is the Lineage in the End?
}

\author{
Kalyani N Bapat ${ }^{1 *}$ and Rajesh B Patil ${ }^{2}$ \\ ${ }^{1}$ Pathologist, CTC, PHO \& BMT Center, Borivali, Mumbai \\ ${ }^{2}$ Hematologist, CTC, PHO \& BMT Center, Borivali, Mumbai
}

\begin{abstract}
According to the World Health Organization (WHO) 2016 classification, acute promyelocytic leukemia (APL) is placed in the category of AML with recurrent cytogenetic abnormalities. Cytogenetic confirmation of reciprocal translocation $t(15: 17)$ is essential criteria for diagnosis of this entity. However, these tests may have a significant time delay and it is usually the characteristic morphology of the abnormal promyelocytes which helps to pick up the diagnosis early and alert the clinician to initiate treatment promptly. The two variants commonly described are the hypergranular and the variant (M3v) forms, but knowledge of other rarer morphological variants of APL becomes crucial in certain situations for the hematopathologist. To highlight this phenomenon, we describe a case report of a 9-year-old female who presented with a hand mirror morphology of the abnormal promyelocytes.
\end{abstract}

Keywords: Acute Promyelocytic Leukemia, Abnormal Promyelocytes, Hand Mirror Morphology, Hypergranular

\section{Introduction}

In the era of multiparametric flow cytometry, cytogenetics and molecular studies, morphological examination still holds an important place in the initial diagnosis of any disorder, especially in resource poor settings. Morphology is the one of the important parameters which helps decide further diagnostic workup and also helps in guiding treatment and hence knowing the morphological variants of a particular entity becomes inevitable. Hand mirror cells have been described mostly but not exclusively in lymphoid malignancies like acute lymphoid leukemias, T- cell lymphomas and multiple myeloma. ${ }^{[1]}$ Nevertheless cases of acute myeloid leukemia with hand mirror cells have also been described in literature often associated with chromosome 13 anomalies. ${ }^{[1]}$ More so very few case reports ${ }^{[1,2,3]}$ have observed this hand mirror morphology in acute promyelocytic leukemia (APL).Considering the urgency with which a case of APL needs to be diagnosed and treated, our case highlights the fact that rare cases of APL can show this morphology and knowledge of this entity will help to alert the hematopathologist early and workup the case faster.

\section{Case Report}

A 9-year-old female child presented with fever, weakness and ecchymotic patches from last 4 days. On examination there was pallor present, however no organomegaly or lymphadenopathy was detected. Laboratory data showed hemoglobin of $72 \mathrm{~g} / \mathrm{L}$, while WBC count was $9.2 \times 10^{9} / \mathrm{L}$ with a platelet count of $17.0 \times 10^{9} / \mathrm{L}$. A detailed peripheral smear examination was done which revealed $60 \%$ atypical cells/ blasts which were of medium size with convoluted nuclear contours some showing cleaved bilobed nuclei and prominent nucleolus (Figure 1, 2). Appreciable amount of cytoplasm was present with few having hypergranulation, however no Auer rod or Faggot cells were seen. In addition to this there was a prominent population of hand mirror shaped atypical cells/blasts. Considering the pediatric age and presence of two types of blast populations, differentials considered were B cell Acute Lymphoblastic leukemia (B-ALL), T cell acute Lymphoblastic leukemia (T-ALL), Mixed phenotype acute leukemia (MPAL) and acute promyelocytic leukemia (APL) Although cleaved nuclear morphology was present no faggot cells were seen. Bone marrow examination was done which also showed similar morphology.

Flow cytometry and cytogenetics with FISH on bone marrow sample was requested on an urgent basis, meanwhile cytochemistry with MPO done showed these cells to be brightly positive for MPO which lowered the possibility of the diagnosis being Acute Lymphoblastic Leukemia. Further flow cytometry analysis revealed that these cells were expressing myeloid markers with bright positivity for cytoplasmic MPO (cMPO), CD13, CD33 and partial positivity for CD34 and CD64, while they were negative for HLA-DR, CD117, CD36, CD10, CD19, CD20, CD2, $\mathrm{CD} 3, \mathrm{CD} 7$, cytoplasmic CD79a and cytoplasmic CD3 thus ruling out possibility of $\mathrm{B}$ or T ALL and MPAL. The bright cMPO positivity and HLA-DR negativity were pointing towards a diagnosis of APL which was further confirmed by interphase FISH which demonstrated the PML-RARA fusion signal in almost 140/200 nuclei (Figure 3).

Baseline screening coagulogram showed $\mathrm{PT}=17.6$ seconds 
(control: 13 secs), aPTT of $42.2 \mathrm{sec}$ (control:28 secs), fibrinogen $=148 \mathrm{mg} / \mathrm{L}$ (Normal range: $150-330 \mathrm{mg} / \mathrm{dl}$ ). Considering the FISH results and as at presentation the WBC count was less than 10,000/ul and platelet count being less than $40,000 /$ cumm, the patient was finally diagnosed as Intermediate risk Acute Promyelocytic Leukemia and was immediately started with ATRA and arsenic trioxide. Coagulopathy was managed with FFP and platelet transfusions. RT-PCR results obtained at a later date also confirmed the fusion transcript. Monitoring of patient was done with daily $\mathrm{CBC}$, peripheral smear examination (PBS) and coagulation parameters for first 15 days. Post stabilization of $\mathrm{CBC}$ and coagulation parameters she was discharged and received further chemotherapy on day care basis. She received induction and consolidation treatment with arsenic trioxide and ATRA as per APL-04 protocol. Currently she is one year post completion of consolidation treatment with negative RT -PCR results at present.

\section{Discussion}

Early diagnosis of APL is essential because of its associated life-threatening coagulopathy and rapid response to ATRA therapy. Although the diagnosis is confirmed by cytogenetic analysis by detection of reciprocal translocation $\mathrm{t}(15: 17)$ the results of these tests are not available immediately. Instead, characteristic cell morphology and cytochemistry offers rapid means of diagnosis wherein treatment can be initiated. Yet rare variations in the typical cell morphology of APL can cause difficulty in diagnosis. A hand mirror variant of microgranular APL was first described by Sun et al in 1991. ${ }^{[2]}$ They had reported a case of microgranular variant of APL wherein the bone marrow showed $80 \%$ of these hand mirror cells some which also contained multiple Auer rods. Cytogenetic studies confirmed it to be $\mathrm{t}(15: 17)$. A similar case has been reported in literature by Sim J and E Ma. ${ }^{[3]}$ They reported 35-year-old Chinese female whose peripheral smear and bone marrow showed atypical cells/ blasts with hand mirror morphology some with cleaved nuclei and distinct nucleolus and strong positivity for MPO. Flow cytometry revealed these atypical cells/ blasts to be CD34 dim positive along with CD13, CD33, cytoplasmic MPO, however they were negative for HLADR, B cell and T cell markers like was seen in our case. Although the morphological appearance was atypical in form of hand mirror shaped cells the bilobed nature of nuclei along with cytochemistry and flow cytometry helped to clinch the diagnosis which was further confirmed by FISH and treatment was initiated early. As per the WHO 2016 classification only two morphological variants of APL have been described namely the hypergranular (typical APL) and the microgranular (M3v) variant. ${ }^{[4]}$ In the year 2000 a committee of morphologists had

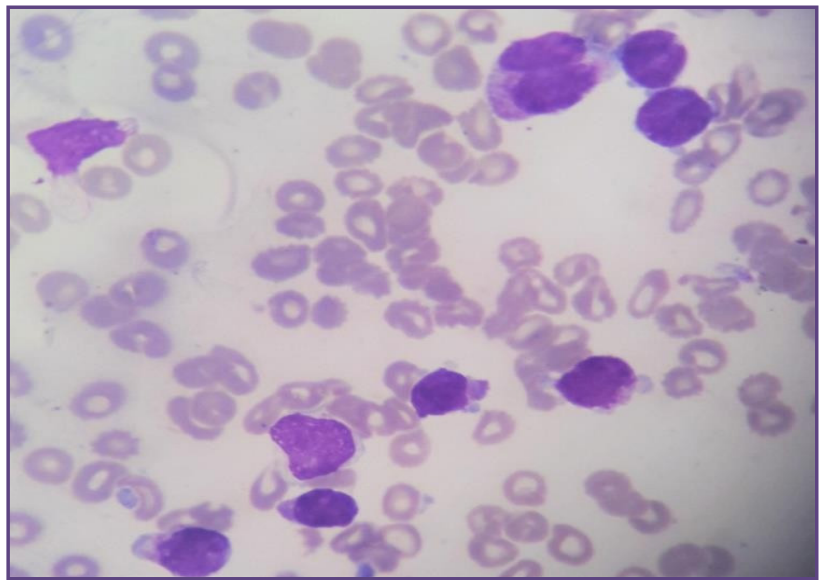

Fig. 1: Peripheral smear image showing abnormal promyelocytes with hand mirror morphology, some with cleaved nuclei (1000x, oil immersion, Leishman stain).

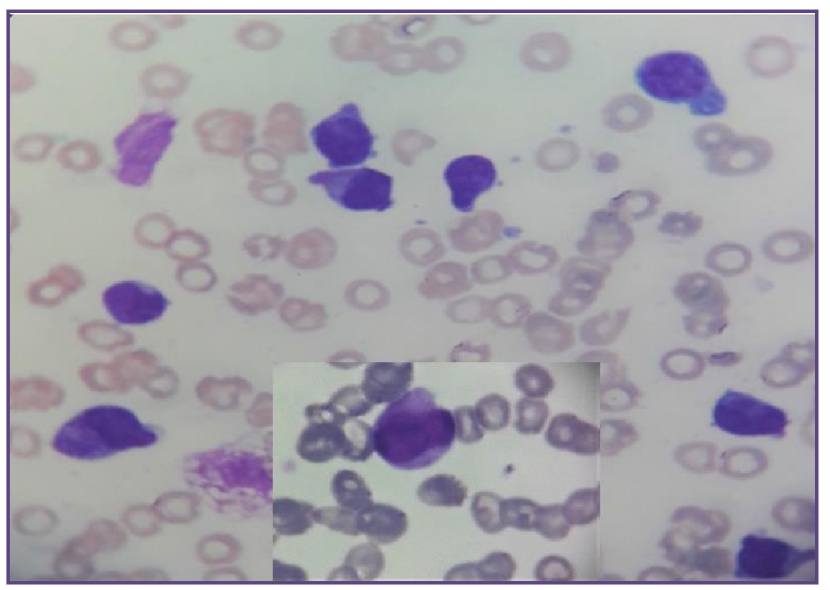

Fig. 2: Peripheral smear image showing abnormal promyelocytes with hand mirror morphology, inset shows bilobed nature of nucleus. $(1000 x$, oil immersion, Leishman stain).

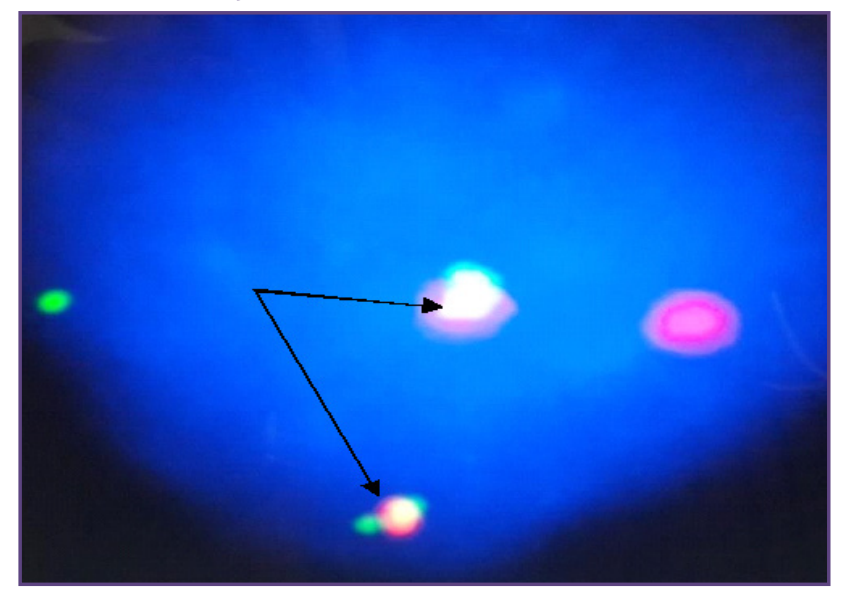

Fig. 3: Dual color dual fusion Interphase FISH showing two yellow fusion signals of PML-RARA and one normal red signal (PML ) and one normal green signal (RARA). 
proposed a new morphological classification of APL which includes classical hypergranular, microgranular (M3v), hyperbasophilic, and acute eosinophilic leukemia with PML-RARA positivity and promyelocytic leukaemia with basophil-like granules. ${ }^{[3]}$ However this classification never gained wide acceptance due to rarity of APL variants other than M3v. Another novel morphological classification system that was found to reliably distinguish APL cases with the $P L Z F / R A R A$ fusion gene from those with ATRAresponsive disease, including those with underlying $P M L /$ $R A R A$ and $N P M / R A R A$ gene rearrangements was proposed at European Workshop in 1997. Application of this 3-parameter classification system (nucleus, granularity, and Auer rods) led to the distinction of 12 categories of cells in APL. ${ }^{(5)}$ Thus, although there is no prognostic implication of diagnosing these other rarer morphological variants, knowing them helps to suspect the disease faster and initiate specific treatment early in the disease course.

\section{Conclusion}

In conclusion although a cytogenetic signature should always be obtained for confirming diagnosis of APL, from a practical point of view attention to other parameters like morphology, cytochemistry and flow cytometry based immunophenotyping can be very useful adjuncts for rapid diagnosis. Knowing rare variants of this disease is of paramount importance which can make the pathologist alert and in turn can alarm the clinician to initiate specific treatment rapidly to decrease the early mortality due to disseminated intravascular coagulation (DIC) which is seen very commonly at presentation of this disease entity.

\section{Funding}

Not applicable

\section{Competing Interests}

Nil

\section{Reference}

1. Sandes AF, Rizzatti EG. Magic mirror in my hand, which is the lineage in the end? A case of acute leukemia with hand mirror cells. Blood 2014; 24 :317.

2. Sun T, Weiss R. Hand mirror variant of microgranular acute promyelocytic leukemia. Leukemia 1991; 5:266-269.

3. Sim J, E Ma. Acute Promyelocytic leukemia or not? Hong Kong Association of Blood transfusion and Hematology . June 2004.

4. Swerdlow SH, Campo E, Harris NL et al. WHO Classification of Tumours of Hematopoietic and Lymphoid Tissues. Lyon: IARC; 2017 Revised 4th edition 134-135.

5. Sainty D, Liso V, Cantù-Rajnoldi A, Head D et al. A new morphologic classification system for acute promyelocytic leukemia distinguishes cases with underlyingPLZF/ RARA gene rearrangements. Blood 2000, 96 (4): 1287-1296

*Corresponding author:

Dr. Kalyani N Bapat, Flat no 204, Sai Prakash Building, Sai Chowk, Barave Road, Khadakpada, Kalyan (West), Thane 421301, Maharashtra, India.

Phone: +919833832452/6381634255

Email: kalyanibapat2812@gmail.com

Date of Submission : 09/09/2020

Date of Revision : $\quad 30 / 12 / 2020$

Financial or other Competing Interests: None. 